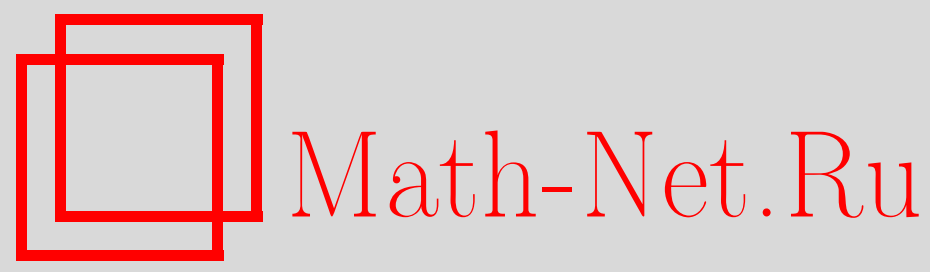

А. А. Коньков, О решениях квазилинейных эллиптических неравенств, обращающихся в нуль в окрестности бесконечности, Матем. заметки, 2000, том 67, выпуск 1, 153-156

DOI: https://doi.org/10.4213/mzm824

Использование Общероссийского математического портала Math-Net.Ru подразумевает, что вы прочитали и согласны с пользовательским соглашением http://www.mathnet.ru/rus/agreement

Параметры загрузки:

IP : 54.174 .149 .18

26 апреля 2023 г., $07: 59: 37$

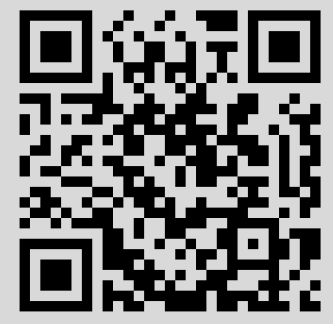




\section{О РЕШЕНИЯХ КВАЗИЛИНЕЙНЫХ ЭЛЛИПТИЧЕСКИХ НЕРАВЕНСТВ, ОБРАЩАЮЩИХСЯ В НУЛЬ В ОКРЕСТНОСТИ БЕСКОНЕЧНОСТИ}

\section{А. А. Коньков}

Пусть $\Omega$ - неограниченное открытое подмножество $\mathbb{R}^{n}, n \geqslant 2, K \subset \mathbb{R}^{n}$ - произвольный, возможно пустой, компакт и $L$ - дифференциальньй оператор вида

$$
L=\sum_{i, j=1}^{n} \frac{\partial}{\partial x_{i}}\left(a_{i j}(x) \frac{\partial}{\partial x_{j}}\right)
$$

где $a_{i j}(x)$ - измеримые функции такие, что $a_{i j} \equiv a_{j i}$ и для некоторой константы $\gamma>0$ при всех $x=\left(x_{1}, \ldots, x_{n}\right) \in \Omega$ и $\zeta=\left(\zeta_{1}, \ldots, \zeta_{n}\right) \in \mathbb{R}^{n}$

$$
\gamma^{-1}|\zeta|^{2} \leqslant \sum_{i, j=1}^{n} a_{i j}(x) \zeta_{i} \zeta_{j} \leqslant \gamma|\zeta|^{2}
$$

Будут изучаться неравенства

$$
(L u-F(x, u)) \operatorname{sign} u \geqslant 0, \quad x \in \Omega \backslash K,
$$

где $F: \Omega \times \mathbb{R} \rightarrow \mathbb{R}$ - локально ограниченная измеримая функция такая, что $F(x,-t)=-F(x, t)$ и $0 \leqslant F(x, t) \leqslant F(x, \tau)$ при всех $0 \leqslant t \leqslant \tau$ и почти всех $x \in \Omega \backslash K$.

Через $Q_{r}$ и $S_{r}$ мы обозначаем соответственно открытый шар $Q_{r}=\{x:|x|<r\}$ и сферу $S_{r}=\{x:|x|=r\}$ радиуса $r$ с центром в точке нуль.

Положим $u_{+}(x)=(u(x)+|u(x)|) / 2, u_{-}(x)=(u(x)-|u(x)|) / 2$ и

$$
M(r)=\operatorname{ess} \sup _{\Omega \cap S_{r}}|u| .
$$

Под решением (1) мы будем подразумевать функцию $u \in W_{2, \mathrm{loc}}^{1}(\Omega \backslash K) \cap L_{\infty, \operatorname{loc}}(\Omega \backslash K)$ такую, что для любой неотрицательной функции $\varphi \in C_{0}^{\infty}(\Omega \backslash K)$

$$
-\int_{\Omega \backslash K} \sum_{i, j=1}^{n} a_{i j}(x) \frac{\partial u_{+}}{\partial x_{i}} \frac{\partial \varphi}{\partial x_{j}} d x \geqslant \int_{\Omega \backslash K} F\left(x, u_{+}\right) \varphi d x
$$

и

$$
\int_{\Omega \backslash K} \sum_{i, j=1}^{n} a_{i j}(x) \frac{\partial u_{-}}{\partial x_{i}} \frac{\partial \varphi}{\partial x_{j}} d x \geqslant-\int_{\Omega \backslash K} F\left(x, u_{-}\right) \varphi d x .
$$

В случае $a_{i j} \in C^{1}(\Omega \backslash K)$ под это определение, бесспорно, подпадает всякая достаточно гладкая функция $u$, удовлетворяющая (1) в классическом смысле.

Мы также пишем

$$
\left.u\right|_{\partial \Omega \backslash K}=0
$$

если $\forall \psi \in C_{0}^{\infty}\left(\mathbb{R}^{n} \backslash K\right) \psi u \stackrel{o}{1} W_{2}(\Omega \backslash K)$.

Работа выполнена при финансовой поддержке Российского фонда фундаментальных исследований, грант № 96-15-96177 школа. 
Таким образом, в случае $\Omega=\mathbb{R}^{n}$ (2) будет заведомо выполнено для любой функции $u \in W_{2, \text { loc }}^{1}(\Omega \backslash K) \cap L_{\infty, \text { loc }}(\Omega \backslash K)$.

Ниже мы будем считать, что $a>0$ и $\sigma>1$ - некоторые фиксированные вещественные числа такие, что $K \subset Q_{a}$, и при этом для измеримой функции $p:[a, \infty) \rightarrow[0, \infty)$ и монотоно не убьвающей функции $g:(0, \infty) \rightarrow(0, \infty)$ при всех $r \in(a, \infty)$ и $t \in(0, \infty)$

$$
p(r) g(t) \leqslant \operatorname{ess} \inf _{x \in \Omega \cap Q_{\sigma r} \backslash\left(Q_{\frac{r}{\sigma}} \cup K\right)} F\left(x, r^{2-n} t\right) .
$$

Из принципа максимума следует, что всякое решение (1), (2) либо удовлетворяет соотношению

$$
M(r) \leqslant M(\rho) \text { при всех } a \leqslant \rho \leqslant r,
$$

либо существует константа $a_{*} \in[a, \infty)$ такая, что при всех $a_{*} \leqslant \rho \leqslant r$

$$
M(r) \geqslant M(\rho) .
$$

Решения (1), (2), для которых имеет место (4), достаточно полно изучены в [1]-[6]. В этом сообщении рассматриваются решения, удовлетворяющие (3).

TEOPEMA 1. Пусть

$$
\int_{0}^{1}(g(t) t)^{-\frac{1}{2}} d t<\infty
$$

$u$

$$
\int_{a}^{\infty} \xi^{n-1} p(\xi) d \xi=\infty
$$

Тогда всякое решение (1), (2), удовлетворяющее условию (3), тохдественно равно нулю в некоторой окрестности $\infty$.

ПримеР 1. Предположим, что $a_{0} \in(0, \infty)$ и $F(x, t)=a_{0}|x|^{s} t \ln ^{\theta}(1 / t)$ для почти всех $x \in \Omega$ и всех достаточно малых положительных $t$. Если $\theta>2$ и $s \geqslant-2$, то применяя теорему 1 , получим, что всякое решение (1), (2), для которого выполнено (3), тождественно равно нулю в некоторой окрестности $\infty$.

Tеорема 2. Пусть имеет место (6) и

$$
\int_{0}^{1}(g(t) t)^{-\frac{1}{2}} d t=\infty
$$

Тогда найдутся постоянные $A>0, B>0$, зависящие только от $n, \gamma, \sigma$ такие, что всякое решение (1), (2), удовлетворяющее условию

$$
\lim _{r \rightarrow \infty} M(r)=0
$$

при почти всех $x$ из некоторой окрестности $\infty$ допускает оченку

$$
|u(x)| \leqslant A|x|^{2-n} H_{0}^{-1}\left(B\left(\int_{a}^{|x|} \xi^{n-1} p(\xi) d \xi\right)^{\frac{1}{2}}\right),
$$

əде

$$
H_{0}(s)=\int_{s}^{1}(g(t) t)^{-\frac{1}{2}} d t,
$$

a $H_{0}^{-1}-$ функиия, обратная $\kappa H_{0}$. 
TEOPEMA 3. Пусть

$$
\int_{a}^{\infty} \min \left\{\xi^{n-1} p(\xi), \xi^{\frac{n-2}{2}} p^{\frac{1}{2}}(\xi)\right\} d \xi=\infty
$$

Тогда найдутся постоянные $A>0, B>0$, зависящие только от $n, \gamma, \sigma$, такие, что всякое решение (1), (2), удовлетворяющее условию (7), либо обращается тождественно в нуль в некоторой окрестности $\infty$, либо при почти всех $x$ из некоторой окрестности $\infty$ допускает оченку

$$
\int_{A|x|^{n-2}|u(x)|}^{1}(g(t) t)^{-\frac{1}{2}} d t+\int_{A|x|^{n-2}|u(x)|}^{1} \frac{d t}{g(t)} \geqslant B \int_{a}^{|x|} \min \left\{\xi^{n-1} p(\xi), \xi^{\frac{n-2}{2}} p^{\frac{1}{2}}(\xi)\right\} d \xi
$$

Теорема 4. Предположим, что имеет место (5) и

$$
\int_{a}^{\infty} \xi^{n-1} p(\xi) d \xi<\infty
$$

Тогда найдутся постоянные $A>0, B>0$, зависящие только от $n, \gamma, \sigma$, такие, что всякое решение (1), (2), удовлетворяющее условию (7), либо обращается тохдественно в нуль в некоторой окрестности $\infty$, либо при всех $r \in[a, \infty)$ допускает оценку

$$
M(r) \geqslant A r^{2-n} G_{0}^{-1}\left(B\left(\int_{r}^{\infty} \xi^{n-1} p(\xi) d \xi\right)^{\frac{1}{2}}\right)
$$

əəe

$$
G_{0}(s)=\int_{0}^{s}(g(t) t)^{-\frac{1}{2}} d t
$$

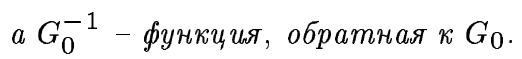

Далее мы будем рассматривать неравенства

$$
\left(L u-c(x)|u|^{\lambda-1} u\right) \operatorname{sign} u \geqslant 0, \quad x \in \Omega \backslash K,
$$

типа Эмдена-Фаулера, где $\lambda \in[0, \infty)$, а измеримая локально ограниченная функция $c: \Omega \rightarrow[0, \infty)$ при всех $r \in[0, \infty)$ подчиняется соотношению

$$
q(r) \leqslant \inf _{x \in \Omega \cap Q_{\sigma r} \backslash\left(Q_{\frac{r}{\sigma}} \cup K\right)} c(x)
$$

для некоторой измеримой функции $q:[a, \infty) \rightarrow[0, \infty)$.

СледСтвиЕ 1. Предположим, что $\lambda<1 u$

$$
\int_{a}^{\infty} \xi^{(2-n) \lambda+n-1} q(\xi) d \xi=\infty
$$

Тогда всякое решение (8), (2), удовлетворяющее условию (3), тождественно равно нулю в некоторой окрестности $\infty$. 
ПримеР 2. Пусть $c(x)=a_{0}|x|^{s}, a_{0} \in(0, \infty)$. Применяя следствие 1 , получим, что при $s \geqslant$ $(n-2) \lambda-n$ и $0 \leqslant \lambda<1$ всякое решение (8), (2), для которого имеет место (3), тождественно равно нулю в некоторой окрестности $\infty$.

Пусть теперь $c(x)=a_{0}|x|^{(n-2) \lambda-n} \ln ^{\varepsilon}|x|, a_{0} \in(0, \infty)$. Тогда при $0 \leqslant \lambda<1$ и $\varepsilon \geqslant-1$ всякое решение (8), (2), удовлетворяющее условию (3), тождественно равно нулю в некоторой окрестности $\infty$.

СлЕДСтвиЕ 2. Предположим, что $\lambda>1$ и при этом выполнено (9). Тогда найдется постоянная $A>0$, зависящая только от $n, \gamma, \sigma, \lambda$, такая, что всякое решение (8), (2), удовлетворяющ,ее условию (7), при почти всех $x$ из некоторой окрестности $\infty$ допускает оченку

$$
|u(x)| \leqslant A|x|^{2-n}\left(\int_{a}^{|x|} \xi^{(2-n) \lambda+n-1} q(\xi) d \xi\right)^{-\frac{1}{\lambda-1}} .
$$

СлЕДСТвИЕ 3. Пусть $\lambda<1 u$

$$
\int_{a}^{\infty} \xi^{(2-n) \lambda+n-1} q(\xi) d \xi<\infty
$$

Тогда существует постоянная $A>0$, зависящая только от $n, \gamma, \sigma, \lambda$, такая, что всякое решение (8), (2), удовлетворяющ,ее условию (7), либо обращается тохсдественно в нуль в некоторой окрестности $\infty$, либо при всех $r \in[a, \infty)$ допускает оценку

$$
M(r) \geqslant A r^{2-n}\left(\int_{r}^{\infty} \xi^{(2-n) \lambda+n-1} q(\xi) d \xi\right)^{\frac{1}{1-\lambda}} .
$$

\section{СПИСОК ЦИТИРОВАННОЙ ЛИТЕРАТУРЫ}

1. Keller J. B. // Comm. Pure Appl. Math. 1957. V. 10. № 4. P. 505-510. 2. Osserman R. // Pacific J. Math. 1957. V. 7. № 4. P. 1641-1647. 3. Vazquez J. L. // Nonlinear Anal. 1981. V. 5. Р. 95-103. 4. Кондратьев В.А., Ландис Е. М. // Матем. сб. 1988. Т. 135(177). № 3. С. 346-360. 5. Кондратьев В. А., Коньков А. А. // Матем. сб. 1994. Т. 185. № 9. С. 81-94. 6. Kon'kov A. A. // Russian J. Math. Phys. 1997. V. 5. № 1. P. 119-122. 